\title{
Management of Diabetes Mellitus in Cushing's Syndrome
}

\author{
Alia Munir John Newell-Price \\ Academic Unit of Diabetes and Endocrinology, University of Sheffield, Sheffield, UK
}

\section{Key Words}

Diabetes $\cdot$ Metformin $\cdot$ Cortisol $\cdot$ Metyrapone $\cdot$

Ketoconazole $\cdot$ Insulin resistance

\begin{abstract}
Active Cushing's syndrome is associated with insulin resistance induced by the high and prolonged circulating level of glucocorticoids. In endogenous Cushing's syndrome the overall incidence of diabetes mellitus and insulin resistance is very likely to be under-reported as not all patients are actively investigated with glucose tolerance tests. Whilst it is common clinical experience that management of diabetes mellitus is necessary in patients with Cushing's syndrome there is a dearth of literature-based evidence to support which regimes are the most effective. Therefore, a pragmatic approach is necessary on an individualized patient basis, whereby patients are stratified according to the severity of their impaired glucose homeostasis. The most effective means of control of diabetes mellitus in a patient with active Cushing's syndrome is to lower the levels of circulating cortisol. This may initially be achieved by using adrenal steroidogenesis blockade with drugs including metyrapone, ketaconazole, or, on occasion, mitotane. The rapid action of metyrapone is particularly suitable in this circumstance. Despite this, diabetes-specific therapy is often necessary and metformin and PPAR- $\gamma$ agonists may be of use, but in the acute setting insulin therapy is frequently needed. Definitive
\end{abstract}

management directed against source driving Cushing's syndrome is often highly effective at either reducing the severity of diabetes, or allowing its complete resolution. Patients experiencing diabetes mellitus in the context of exogenously administered glucocorticoids may well require insulin therapy for the period that the high levels of steroids are being administered. Despite resolution of Cushing's syndrome after definitive treatment patients may continue to exhibit insulin resistance. This and other cardiovascular risk factors require ongoing and long-term attention.

Copyright $\odot 2010$ S. Karger AG, Basel

\section{Overview}

Endogenous Cushing's syndrome is uncommon, but glucocorticoids are a very frequently prescribed medication, and if taken for long enough at sufficient doses Cushing's syndrome may result. Therefore, the metabolic consequences of Cushing's syndrome are common. The predominant effect of glucocorticoids on glucose homeostasis is that of insulin resistance, but there are also data suggesting a decrease in insulin secretion. There are, however, few data on the prevalence of diabetes mellitus in Cushing's syndrome, and fewer still on the best means for management. Here, we focus on what is known of the prevalence of diabetes mellitus in Cushing's syndrome and then we offer practical guidelines for management.

\section{KARGER}

Fax +4161306 1234

E-Mail karger@karger.ch

www.karger.com
(C) 2010 S. Karger AG, Basel

0028-3835/10/0925-0082\$26.00/0

Accessible online at:

www.karger.com/nen
J. Newell-Price

Academic Unit of Diabetes and Endocrinology, University of Sheffield

Room OU142, Floor O, Royal Hallamshire Hospital

Sheffield (UK)

Tel. +44 114226 1409, Fax +44 114271 1863, E-Mail j.newellprice@ sheffield.ac.uk 


\section{Concerns over the Effects of Exogenous Glucocorticoids}

Glucocorticoids are frequently administered for inflammatory conditions, especially inflammatory arthritic conditions. Recent data have highlighted the concerns of patients and non-diabetes physicians with respect to developing diabetes mellitus whilst taking glucocorticoids: a survey of 115 patients in Europe revealed that diabetes ranked second only to worries over osteoporosis, whilst for the 114 physicians treating rheumatic conditions who were questioned, developing diabetes ranked as their greatest concern [1]. These data reinforce the need to actively identify and manage the metabolic consequences of exogenous glucocorticoids, but there are no data as to the optimum means of achieving this.

\section{Exogenous Glucocorticoids and Prevalence of Diabetes Mellitus}

The precise prevalence and risk of developing diabetes mellitus when taking exogenous glucocorticoids is not established. Part of this relates to the predominant effect of insulin resistance, and this may be disclosed more fully on oral glucose tolerance testing. The problem lies in the fact that glucose tolerance testing is rarely performed in this large patient cohort. The incidence of diabetes in those on glucocorticoids has been estimated in few studies. A population of 644,495 in the primary care setting revealed a group of 2,647 on glucocorticoid therapy who were followed for 1 year and the incidence of diabetes compared to a control group of 5,294 subjects [2]. A cumulative dose equivalent of hydrocortisone $>2.5 \mathrm{~g}$ was associated with a relative risk for diabetes of 1.25 (odds ratio, 1.01-1.54). In practical terms this is the anti-inflammatory equivalent of prednisolone $10 \mathrm{mg}$ /day for 2 months. Furthermore, a recent meta analysis revealed an odds ratio of 1.5-2.5 for a new diagnosis of diabetes when taking exogenous glucocorticoids, whilst highlighting that fasting plasma glucose will underestimate disease compared to a glucose tolerance test [3].

\section{Prevalence of Diabetes in Endogenous Cushing's Syndrome}

The prevalence of diabetes in endogenous Cushing's syndrome has been assessed in very few studies, as illustrated in table 1 [4-7]. Overall, approximately $40-45 \%$ of
Table 1. Prevalence of diabetes mellitus (DM) and glucose intolerance (IGT) in endogenous Cushing's syndrome

\begin{tabular}{lrrll}
\hline & & $\mathrm{n}$ & $\% \mathrm{DM}$ & $\% \mathrm{IGT}$ \\
\hline Friedman et al. [4] & 1996 & 16 & 37.5 & 12.5 \\
Biering et al. [5] & 2000 & 131 & 40.5 & 28 \\
Pecori Giraldi et al. [6] & 2003 & 280 & 44 & \\
Manicini et al. [7] & 2004 & 49 & 47 & 21 \\
& & & & $(64 \% \mathrm{FBG}<6.1 \mathrm{mmol} / \mathrm{l})$
\end{tabular}

FBG $=$ Fasting blood glucose

patients have diabetes mellitus, whilst approximately 10 $30 \%$ have impaired glucose tolerance. Importantly, the data show the short comings of using fasting plasma glucose to assess glucose homeostasis in Cushing's syndrome since $64 \%$ of the $47 \%$ of 49 patients who had diabetes according to the oral glucose tolerance test had a fasting plasma glucose of $<6.1 \mathrm{mmol} / \mathrm{l}$ [7]. This finding is not unexpected in light of the predominant mechanism of insulin resistance, but underlies the need for adequate clinical assessment.

In general, the level of hypercortisolaemia correlates with the presence of insulin resistance and diabetes as evidenced by an association with higher levels of UFC [4, $7]$, but this was not assessed or found in all studies $[5,6]$. This discrepancy is not surprising as there is wide interindividual susceptibility to the adverse effects of glucocorticoids and careful studies have also revealed wide intra-individual variability when assessing glucocorticoid sensitivity in different tissues. Moreover, there appears to be an element of 'imprinting' of the metabolic effects of hypercortisolaemia with impaired glucose tolerance being evident up to 5 years after remission of Cushing's syndrome [8].

\section{Management of Diabetes in Cushing's Syndrome}

For both exogenous and endogenous Cushing's syndrome there is a dearth of evidence as to optimum treatment regimens, and as such recommendations are largely based on current best practice.

Diabetes Associated with Exogenous Glucocorticoids

Prolonged exposure to low doses of glucocorticoids, or shorter exposure to high doses, should be anticipated to provoke the onset of diabetes, or at least insulin resistance 


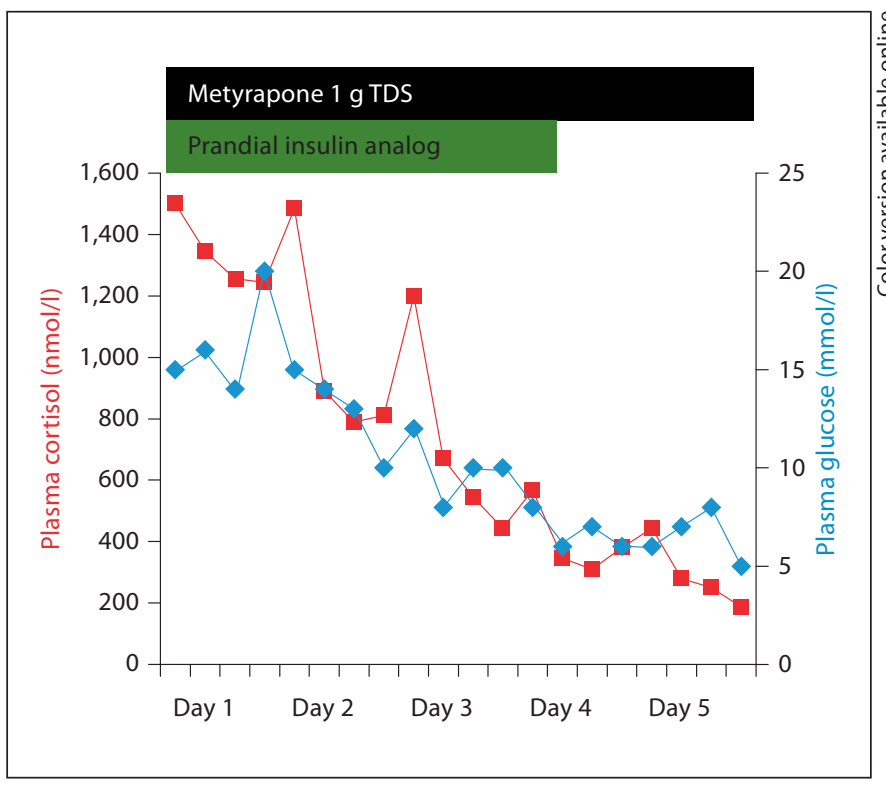

Fig. 1. Effect of acutely lowering cortisol and use of insulin analog to control diabetes mellitus in a patient with Cushing's disease due to macroadenoma. Note that plasma glucose control is maintained as cortisol levels improve following use of metyrapone.

and other features of the metabolic syndrome. To date there is no study that has assessed directly the time taken to develop evidence of metabolic complications, such as fatty liver. Clinical experience is that high doses of dexamethasone, as used in neurosurgical settings, are frequently associated with short-term hyperglycaemia and in the acute hospital setting this may need treatment with insulin analogs or insulin infusions. In the more chronic scenario in the community, especially where lower doses of glucocorticoids are being administered, metformin may be sufficient to prevent or alleviate symptoms of hyperglycaemia and control long-term HbAlc levels. If this fails, then insulin or insulin analogs may be needed.

\section{Diabetes Associated with Endogenous Cushing's Syndrome}

In endogenous Cushing's syndrome management may be divided into the acute setting where hyperglycaemia needs immediate management, for example before urgent definitive surgery, and the chronic setting where hyperglycaemia may prevail, e.g. in the incompletely controlled hypercortisolaemia after pituitary radiotherapy for Cushing's disease.

In the acute setting, the priority is to achieve rapid control. Plasma glucose often improves rapidly or is eas-

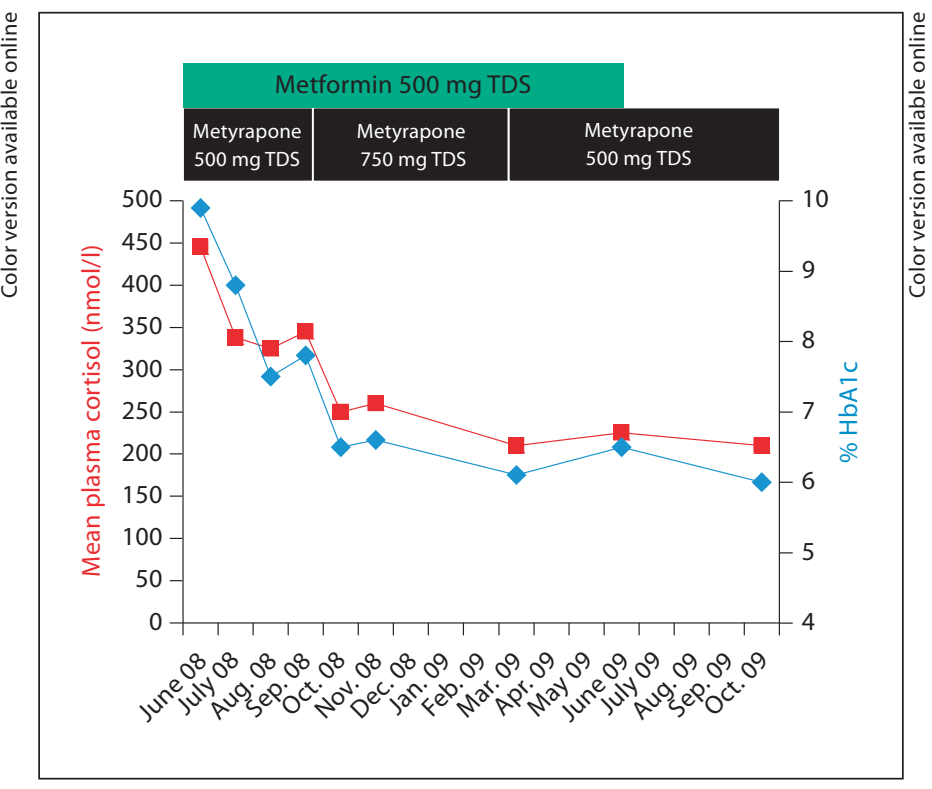

Fig. 2. Chronic management of diabetes mellitus in a patient with Cushing's disease following radiotherapy. Note control of HbAlc even after withdrawal of metformin once plasma cortisol is controlled. ier to control within days to weeks of instigation of cortisol-lowering therapy. Because of its rapid onset of action, metyrapone is especially suited to this use. In addition to this, prandial insulin analogs or infusions of insulin may be needed if the patient is acutely unwell. An example is shown in figure 1 .

In the chronic setting the priority is to control symptoms and achieve long-term control as reflected by HbAlc levels. Here, oral agents, especially metformin with its action improving insulin resistance, may suffice in combination with cortisol-lowering agents as needed. Nevertheless, some patients may need insulin or insulin analogs and others may achieve adequate control through the use of other agents such as sulphonylureas and PPAR- $\gamma$ agonists. There are no data on the use of other newer agents, such as GLP-1 agonists. In the chronic setting close monitoring is needed as cortisol levels fall, e.g. after pituitary radiotherapy or in cyclical disease, as there may often be the need for reduced dosage of hypoglycaemic agents, and increased risk of hypoglycaemia. Moreover, in the undiagnosed or newly diagnosed patient with Cushing's syndrome presenting with diabetes variations in insulin requirements may be a clear pointer to cyclical disease. An illustration of management in the chronic setting is shown in figure 2 . 


\section{Future Avenues for Research}

The long-term effects of hypercortisolaemia need more investigation. The few studies that have been performed in endogenous Cushing's syndrome in this area have important implications for the huge number of patients treated with glucocorticoids. One very important area is to gain a greater understanding of the metabolic, cardiovascular and bone effects of different glucocorticoids as the often quoted 'dose equivalents' are based on anti-inflammatory effects rather than metabolic effects. Insight can be gained from clinical experience of the effects of dexamethasone rather than hydrocortisone in the treatment of congenital adrenal hyperplasia, where all too frequently 'equivalent' doses of long-term dexamethasone are associated with significant metabolic side ef- fects. This is further illustrated by data in a few patients studied in a cross-over design that demonstrated a trend to a worse effect from dexamethasone despite 'a dose equivalent', when used for adrenal replacement [9]. What is needed are robust adequately powered studies that take into account inter-individual variation of glucocorticoid sensitivity [10] and an analysis of the non-inflammatory effects of glucocorticoids. Similar studies are needed for endogenous Cushing's syndrome and this will be facilitated by large collaborative databases.

\section{Disclosure Statement}

The authors have no conflicts of interest to declare.

\section{References}

1 Van der Goes MC, Jacobs JW, Boers M, et al: Patients' and rheumatologists' perspectives on glucocorticoids an exercise to improve the implementation of the EULAR recommendations on the management of systemic glucocorticoid therapy in rheumatic diseases. Ann Rheum Dis 2010;69:1015-1021.

-2 Gulliford MC, Charlton J, Latinovic R: Risk of diabetes associated with prescribed glucocorticoids in a large population. Diabetes Care 2006;29:2728-2729.

- 3 Clore JN, Thurby-Hay L: Glucocorticoid-induced hyperglycemia. Endocr Pract 2009;15: 469-474.

- 4 Friedman TC, Mastorakos G, Newman TD, Mullen NM, Horton EG, Costello R, Papadopoulos NM, Chrousos GP: Carbohydrate and lipid metabolism in endogenous hypercortisolism: shared features with metabolic syndrome X and NIDDM. Endocr J 1996;43: 645-655.
5 Biering H, Knappe G, Gerl H, Lochs H: Prevalence of diabetes in acromegaly and Cushing syndrome. Acta Med Austriaca 2000;27: $27-31$.

-6 Pecori Giraldi F, Moro M, Cavagnini F, Study Group on the Hypothalamo-Pituitary-Adrenal Axis of the Italian Society of Endocrinology: Gender-related differences in the presentation and course of Cushing's disease. J Clin Endocrinol Metab 2003;88: 1554-1558.

7 Mancini T, Kola B, Mantero F, Boscaro M, Arnaldi G: High cardiovascular risk in patients with Cushing's syndrome according to $1999 \mathrm{WHO} / \mathrm{ISH}$ guidelines. Clin Endocrinol (Oxf) 2004;61:768-777. $\checkmark 8$ Colao A, Pivonello R, Spiezia S, Faggiano A, Ferone D, Filippella M, Marzullo P, Cerbone G, Siciliani M, Lombardi G: Persistence of increased cardiovascular risk in patients with Cushing's disease after five years of successful cure. J Clin Endocrinol Metab 1999; 84:2664-2672

-9 Suliman AM, Freaney R, Smith TP, McBrinn Y, Murray B, McKenna TJ: The impact of different glucocorticoid replacement schedules on bone turnover and insulin sensitivity in patients with adrenal insufficiency. Clin Endocrinol (Oxf) 2003;59:380-387.

10 Ebrecht M, Buske-Kirschbaum A, Hellhammer D, Kern S, Rohleder N, Walker B, Kirschbaum C: Tissue specificity of glucocorticoid sensitivity in healthy adults. J Clin Endocrinol Metab 2000;85:3733-3739. 